\title{
Low-dose anticholinergic therapy causes cognitive impairment in Parkinson's disease patients
}

\author{
${ }^{* 1}$ Olga Gavriliuc, ${ }^{2}$ Alexandru Andrusca, ${ }^{3}$ Lilian Popil, ${ }^{3}$ Mihail Gavriliuc \\ ${ }^{1}$ Scientific Laboratory of Functional Neurology, Diomid Gherman Institute of Neurology and Neurosurgery \\ ${ }^{2}$ Department of Neurosurgery, ${ }^{3}$ Department of Neurology \\ Nicolae Testemitanu State University of Medicine and Pharmacy, Chisinau, the Republic of Moldova
}

Authors' ORCID iDs, academic degrees and contributions are available at the end of the article

${ }^{\star}$ Corresponding author - Olga Gavriliuc, e-mail: olgagavriliuc@yahoo.com

Manuscript received July 29, 2021; revised manuscript October 03, 2021; published online October 12, 2021

\begin{abstract}
Background: Before L-Dopa's discovery, anticholinergic drugs were among the first treatments for Parkinson's disease. Only now trihexyphenidyl (THP) is approved to treat unresponsive L-dopa tremors in young, cognitively unaffected Parkinson's disease patients. However, there are no specific recommendations for disease duration, medication dose, or cognitive status. In low-income countries, THP is still frequently used in Parkinson's disease patients with tremor. The objective of the current study was to evaluate cognitive performance in Parkinson's disease patients receiving a low dose of THP. Material and methods: The study was performed on nineteen PD patients, nine of whom were on THP. All patients completed MoCA cognitive assessment. The patients were matched depending on their age, disease severity based on UPDRS III and duration of the disease.

Results: The THP patients were taking an average dose of $3.3 \mathrm{mg}$ of THP daily for an average of 1.8 years. There were no statistical differences between THP patients and non-THP patients in age (64.8 \pm 4.8 vs 67.2 \pm 6.9 , $\mathrm{p}=0.4)$, UPDRS III ( $32.1 \pm 8.9$ vs $41.5 \pm 20.6$, $\mathrm{p}=0.2)$ and disease duration $(6.2 \pm 4.9$ vs 7.0 $\pm 4.0, \mathrm{p}=0.7)$. The THP patients had lower cognitive performance, with a total MoCA of $19.22 \pm 3.3$ vs. non-THP patients $24.2 \pm 3.0, \mathrm{p}=0.003$.

Conclusions: In Parkinson's disease patients, even a low dose of THP causes significant cognitive loss.

Key words: Parkinson's disease, cognition, dementia, anticholinergic.
\end{abstract}

\section{Cite this article}

Gavriliuc O, Andrusca A, Popil L, Gavriliuc M. Low-dose anticholinergic therapy causes cognitive impairment in Parkinson's disease patients. Mold Med J. 2021;64(4):66-68. https://doi.org/10.52418/moldovan-med-j.64-4.21.12.

\section{Introduction}

Parkinson's disease is one of the most common neurodegenerative diseases. Around the world, estimates of Parkinson's disease (PD) incidence range from 5 to 35 new cases per 100000 individuals per year [1]. In recent years, it has become apparent that PD has a wide range of non-motor features, including autonomic dysfunction, sleep disorders, hyposmia, and, as well as cognitive impairment [2-4].

Progressive degeneration of dopaminergic neurons in substantia nigra pars compacta is known to be the major neuropathological feature of PD. For the past 50 years, the most effective symptomatic treatment has been the administration of dopaminergic replacement drugs, especially levodopa [5]. However, later was observed that there are many symptoms of PD that do not respond to levodopa, such as axial symptoms, cognitive impairment or dementia [6]. In this context, recent evidence suggests that the degeneration of adrenergic, serotonergic, glutamatergic and cholinergic neurons also plays an important role [7].

According to recent studies, approximately $36 \%$ of PD patients develop dementia after 4 years of follow-up, and 83 percent have dementia after 20 years of follow-up, which is thought to be due to cholinergic deficiency [8]. As a result, cholinergic deficiency plays an important role in the pathophysiology and bio-cellular mechanisms of Parkinson's disease [9]. Cholinergic neurons, which are widely distributed throughout the brain parenchyma, play an important role in a variety of brain processes, including cognitive functions [6, 7]. According to a recent study, patients with dementia-free Parkinson's disease had moderate cholinergic dysfunction, whereas those with dementia associated with Parkinson's disease had severe cholinergic deficiency in various cortical regions [10].

One of the first Parkinson's disease treatments were anticholinergic drugs [11]. Currently, anticholinergics are approved for unresponsive L-dopa tremors in young and cognitively unaffected patients and because of a low price it is still widely used in low-income countries [12]. Regardless, a relevant issue arises because people with Parkinson's disease may have a subclinical cholinergic deficiency and the use of anticholinergic drugs may result in faster cognitive deterioration due to cholinergic system suppression, as well as a relatively unknown risk, the risk of long-term drug dependence. The goal of this study was to evaluate cognitive performance in Parkinson's disease patients taking a low 
dose of trihexyphenidyl (THP) and patients who did not take any anticholinergic medications.

\section{Material and methods}

The study included 19 patients divided into two groups: 9 patients with Parkinson's disease who received trihexyphenidyl daily and 10 patients with Parkinson's disease who did not receive trihexyphenidyl at all. The two groups of patients were similar in terms of age and disease severity. The Unified Parkinson's Disease Rating Scale (UPRDS) part III was used to assess disease severity, and the Montreal Cognitive Assessment Test (MOCA) was used to compare cognitive status.

The statistical analysis was performed using SPSS, version 23.0. Data are expressed by mean and standard deviation in the case of normal distribution and median with IQR for variables that are non-normally distributed. The differences between the two groups of patients were analyzed with the $\mathrm{T}$ or Mann-Whitney test, as appropriate. In all analyses, $\mathrm{p}$ values $<0.05$ were considered significant.

The current research is a part of a larger project for research on PD that was approved by the Research Ethics Committee of Nicolae Testemitanu State University of Medicine and Pharmacy (protocol No 44 of 12.04.2018).

\section{Results}

Patients were divided into two groups: 9 Parkinson's disease patients who received trihexyphenidyl (THP group) were compared to 10 Parkinson's disease patients who did not receive trihexyphenidyl (non-THP group). Patients in the THP group received this medication for an average of 1.8 years, at a daily dose of $3.3 \mathrm{mg}$ THP. The patients in the two groups did not differ in terms of age, disease duration, disease severity, as measured by UPDRS III. Patients' cognitive ability, as measured by the MoCA test, was statistically significant between patient groups (tab. 1).

Question number 3 of the MoCA test (attention) showed the largest statistical difference, so that patients in the THP group had an average value of $1.56 \pm 0.5$ compared to patients in the non-THP group, who had an average value of $2.7 \pm 0.5$, so the difference is statistically significant, $\mathrm{p}=0.001$. Among the separate items of UPDRS III only the kinetic tremor of the left upper limb was significantly different between both groups $(p=0.04)$, patients in the non-THP group being more affected.

Table 1. Demographic and clinical characteristics of both patients' groups

\begin{tabular}{|l|c|c|c|}
\hline & THP $(\mathbf{n}=9)$ & non-THP $(\mathbf{n = 1 0 )}$ & $\mathbf{p}$ \\
\hline Age (years) & $64.8 \pm 4.8$ & $67.2 \pm 6.9$ & 0.4 \\
\hline Disease duration (years) & $6.2 \pm 4.9$ & $7.0 \pm 4.0$ & 0.5 \\
\hline UPDRS III & $32.1 \pm 8.9$ & $41.5 \pm 20.6$ & 0.2 \\
\hline MoCA total & $20(5)$ & $25(6)$ & 0.003 \\
\hline
\end{tabular}

THP - group of patients administering trihexyphenidyl; non-THP - group of patients who did not administer trihexyphenidyl. Data are presented as mean \pm standard deviation for all except marked data with ${ }^{*}$, presented as median (IQR). The value of $\mathrm{p}$ was calculated with the student $t$ test, except for the marked data with ${ }^{*}$, which was calculated with the Mann-Whitney test due to abnormal data distribution. UPDRS III - the motor part of the Parkinson's disease disability scale. MoCA - Montreal cognitive assessment.

\section{Discussion}

There's no doubt that PD causes cognitive impairment, which leads to dementia and a higher mortality and morbidity rate. At the ten-year follow-up, $60 \%$ of patients are diagnosed with dementia; at the twenty-year over $83 \%$ are diagnosed with the disease. Anticholinergic medications diminish attentional function, which is most typically associated with alertness, and causes recent memory problems [8, 13]. Therefore, even if anticholinergics provide a benefit in terms of motor function, there is a high chance that the patients may acquire a cognitive issue earlier in the course of the disease. We found in this study that even a small daily dose of THP leads to a worse cognitive outcome.

In patients with $\mathrm{PD}$, the density of cholinergic neurons is reduced in all cortical areas [14]. Degeneration of cholinergic neurons, however, is only one of the mechanisms underlying cognitive impairment, according to recent studies $[7,9]$. Previous clinical observations with anticholinergic drugs, which were the first symptomatic treatment for patients with PD before the discovery of levodopa, led to the hypothesis that both cholinergic and dopaminergic signalling systems must be in balance for the striatum to function normally in movement control [11]. Anticholinergic medicines are thought to attenuate the increased striatal cholinergic tone generated by the loss of dopamine in PD patients, restoring the balance between these two signalling systems to some extent [6]. However, it is still unclear whether and how cholinergic disorders contribute to motor and non-motor symptoms. The broad degradation of cholinergic neurons in most patients with PD causes cognitive deficits at some point in the disease and long-term THP treatment has the potential to cause irreversible alterations in the brain, according to the findings of experimental studies on mice [15].

The prefrontal cortex and the frontal basal nuclei, which are severely affected by cholinergic deficiency, are also implicated in the control of gait. Gait and balance can only be coordinated with cognitive function $[16,17]$. As a result, THP could affect independence and quality of life by exacerbating gait and balance problems.

Additionally, THP use is often accompanied by physical symptoms, such as dilated pupils, flushed skin, constipation, as well as rapid heart rate and ataxia [18]. Giving up this drug is extremely difficult, especially if a cognitive deficit has already developed. International guidelines recommend the use of THP only in young PD patients, without cognitive impairment, who have a low risk of developing dementia. 
However, younger patients have a higher risk of developing drug abuse with THP.

\section{Conclusions}

As a result, it can be concluded that there are no clear data on the age of individuals who would benefit and what amount of THP would be effective for the treatment of PD motor symptoms without causing irreversible effects. No doubt this is a serious public health concern because THP is commonly used in the Republic of Moldova, and chronic THP use causes cognitive deterioration, dependence, and abuse even in a low dose.

\section{References}

1. Marek K, Jennings D, Lasch S, et al. The Parkinson Progression Marker Initiative (PPMI). Prog Neurobiol. 2011;95(4):629-635. https://doi. org/10.1016/j.pneurobio.2011.09.005.

2. Bosboom JL, Stoffers D, Wolters EC. Cognitive dysfunction and dementia in Parkinson's disease. J Neural Transm. 2004;111(10-11):1303-1315. https://doi.org/10.1007/s00702-004-0168-1.

3. Poewe W, Seppi K, Tanner CM, Halliday GM, Brundin P, Volkmann J, Schrag AE, Lang AE. Parkinson's disease. Nat Rev Dis Prim. 2017;3:1-21. https://doi.org/10.1038/nrdp.2017.13.

4. Ascherio A, Schwarzschild MA. The epidemiology of Parkinson's disease: risk factors and prevention. Lancet Neurol. 2016;15(12):1257-1272. https://doi.org/10.1016/S1474-4422(16)30230-7.

5. Carranza M, Snyder MR, Davenport Shaw J, Zesiewicz TA. Parkinson's disease: a guide to medical treatment. Torino: SEEd; $2013.354 \mathrm{p}$.

6. Hilker R, Thomas AV, Klein JC, Weisenbach S, Kalbe E, Burghaus L, et al. Dementia in Parkinson's disease: functional imaging of cholinergic and dopaminergic pathways. Neurology. 2005;65(11). https://doi. org/10.1212/01.wnl.0000191154.78131.f6

7. Ztaou S, Amalric M. Contribution of cholinergic interneurons to striatal pathophysiology in Parkinson's disease. Neurochem Int. 2019;126:1-10. https://doi.org/10.1016/j.neuint.2019.02.019.

8. Williams-Gray CH, Evans JR, Goris A, Foltynie T, Ban M, Robbins TW, Brayne C, Kolachana BS, Weinberger DR, Sawcer SJ, Barker RA. The distinct cognitive syndromes of Parkinson's disease: 5 year follow-up of the CamPaIGN cohort. Brain. 2009;132(Pt 11):2958-2969. https://doi. org/10.1093/brain/awp245.

9. Tanimura A, Pancani T, Lim SAO, Tubert C, Melendes AE, Shen W, Surmeier DJ. Striatal cholinergic interneurons and Parkinson's disease. Eur J Neurosci. 2018 May;47(10):1148-1158. doi: 10.1111/ejn.13638.

10. Gomperts SN. Lewy body dementias: dementia with Lewy bodies and Parkinson's disease dementia. Continuum (Minneap Minn). 2016;22:435-463. doi: 10.1212/CON.0000000000000309.

11. Pillon B, Dubois B, Duchesne N, Masson H. Acute and long-term administration of anticholinergics in Parkinson's disease: specific effects on the subcortico-frontal syndrome. Brain Cogn. 1999;40(2):289-313. doi: 10.1006/brcg.1999.1083.

12. Popil L, Gavriliuc O, Rotaru L, Gavriliuc M. Efectele tratamentului cu Trihexifenidil asupra funcției cognitive la pacienții cu boala Parkinson [Effects of trihexyphenidil treatment on cognitive function in patients with Parkinson's disease: review article]. [Bull Acad Sci Moldova]. 2020;(3):27-33. Romanian.

13. Sasikumar S, Strafella AP. Imaging mild cognitive impairment and dementia in Parkinson's disease. Front Neurol. 2020;11:1-8. https://doi. org/10.3389/fneur.2020.00047.

14. Deffains M, Adler A, Joshua M, Goldberg JA. Basal ganglia : acetylcholine interactions and behavior. In: Reference module in neuroscience and biobehavioral psychology. Elsevier; 2017. https://doi.org/10.1016/ B978-0-12-809324-5.02520-7.

15. Tanda G, Ebbs AL, Kopajtic TA, Elias LM, Campbell BL, Newman AH, Katz JL. Effects of muscarinic M1 receptor blockade on cocaine- induced elevations of brain dopamine levels and locomotor behavior in rats. J Pharmacol Exp Ther. 2007;321(1):334-344. https://doi.org/10.1124/ jpet.106.118067.

16. Gavriliuc O, Andrusca A, Gavriliuc M. Clinical indicators of gait freezing in Parkinson's disease. Mold Med J. 2020;63(2):31-33. https://doi. org/10.5281/zenodo.3866002.

17. Morris R, Martini DN, Madhyastha T, Kelly VE, Grabowski TJ, Nutt J, Horak F. Overview of the cholinergic contribution to gait, balance and falls in Parkinson's disease. Parkinsonism Relat Disord. 2019;63:20-30. https://doi.org/10.1016/j.parkreldis.2019.02.017.

18. Torrents R, Ferré JF, Konareff A, Hemery P, Sherwin K, Lassalle C, Simon N, Scerra S. Misuse of Trihexyphenidyl (Artane) on Réunion Island. J Clin Psychopharmacol. 2018;38(3):250-253. https://doi.org/10.1097/ JCP.0000000000000882.

\section{Authors' ORCID iDs and academic degrees}

Olga Gavriliuc, MD, Phd Applicant - https://orcid.org/0000-0003-0677-5467

Alexandru Andrusca, MD, Phd Applicant - https://orcid.org/0000-0001-6174-7114

Lilian Popil, MD, Undergradute Student - https://orcid.org/0000-0002-8227-0666

Mihail Gavriliuc, MD, PhD, Professor of Neurology - https://orcid.org/0000-0002-5789-2842

\section{Authors' contribution}

OG and AA drafted the first manuscript and analyzed the data, LP collected the data, MG designed the trial and revised the manuscript critically. All the authors revised and approved the final version of the manuscript.

\section{Funding}

The study was supported by Diomid Gherman Institute of Neurology and Neurosurgery and Nicolae Testemitanu State University of Medicine and Pharmacy. The authors are independent and take responsibility for the integrity of the data and accuracy of the data analysis.

\section{Ethics approval and consent to participate}

The research was approved by the Research Ethics Committee of Nicolae Testemitanu State University of Medicine and Pharmacy (protocol No 44 of April 12, 2018).

\section{Conflict of Interest}

The authors have no conflict of interests to declare. 